\title{
Simultaneous Optimisation of Confocal and Non-Confocal Images in an AOSLO with a Reconfigurable Aperture Pattern
}

\author{
Biswajit Pathak ${ }^{10000-0001-6168-2111]}$, Laura Young ${ }^{1,2[0000-0002-1805-3376]}$, and \\ Hannah Smithson ${ }^{1[0000-0001-8904-8315]}$ \\ 1 University of Oxford, Oxford OX2 6GG, UK \\ 2 Newcastle University, Newcastle NE2 4HH, UK \\ https://www.psy.ox.ac.uk/research/perception-lab/ \\ high-resolution-retinal-imaging \\ \{biswajit.pathak, laura.young, hannah.smithson\}@psy.ox.ac.uk
}

\begin{abstract}
The conventional adaptive optics scanning laser ophthalmoscopy (AOSLO) arrangement is specifically designed to capture the confocal (directly backscattered) light by placing a physical pinhole conjugate to a chosen layer in the retina. This arrangement can be used to generate high contrast images of the photoreceptor mosaic by limiting the light from other retinal layers, such as the retinal pigment epithelium. However, there is growing demand for the study of different retinal features that has led to the development of different off-axis techniques to collect the non-confocal (multiply scattered) light. In this paper, we replace the physical pinhole of the AOSLO with a reconfigurable aperture to simultaneously collect the directly backscattered light, generating confocal images, as well as the multiply scattered light, generating non-confocal images. The reconfigurable aperture pattern is implemented with a digital micromirror device (DMD) and is optimised based on the information collected from Shack Hartmann wavefront sensor data. We present preliminary experimental results with a human eye to illustrate our findings.
\end{abstract}

Keywords: Adaptive optics imaging - Retinal imaging · Non-confocal imaging · Digital micromirror device.

\section{Introduction}

The application of adaptive optics in retinal imaging has enabled visualisation of the retina with cellular resolution $[1,2,3]$. The adaptive optics scanning laser ophthalmoscope (AOSLO) can generate images of retinal substructures at the cellular level such as cone photoreceptors. A focussed spot is scanned across the human retinal surface and a detector is used to capture the directly back scattered (confocal) light at every scan position. The out-of-focus light from other retinal layers is eliminated by placing in front of the detector a pinhole that is co-aligned with, and at a plane conjugate, to the focussed spot on the retina $[1,4]$. 
The need to study other retinal features such as retinal pigment epithelial cells, which are weakly reflecting, paved the way for several non-confocal techniques [5] such as; darkfield [6], split-detection [7] and multi-offset techniques [8]. All of these techniques involve the use of an offset aperture that blocks the directly backscattered light, thereby enabling the detection of multiply scattered light. In these techniques, the multiply scattered light is collected by separating it from the confocal light with either an opaque filament [6], annular circular mask [7] or laterally displaced pinhole $[8,9]$. However, most of these configurations make use of a physical pinhole to collect the multiply scattered light and cannot be reconfigured or realigned in real time.

Recently, the limitations imposed by a physical aperture were addressed to some extent by using a dynamically reconfigurable aperture pattern implemented with a digital micromirror device (DMD) [10]. This arrangement optimised the orientation of the reconfigurable aperture pattern to explore the contrast enhancement of the retinal microvasculature in split-detection imaging.

In the present work, both confocal as well as non-confocal (darkfield) imaging configurations are optimised simultaneously using a reconfigurable aperture pattern. The optimisation is based on the information collected from Shack Hartmann $(\mathrm{SH})$ wavefront sensor data and is realised by exploiting the programmable facility of the DMD. Experimental results are included from a human participant, demonstrating simultaneous optimisation of confocal and darkfield images.

\section{Methodology}

In this section, we explain the working principle of a quality metric that helps in the estimation of pupil diameter. The intensity distributions of the SH spots are used to estimate the pupil diameter and the SH slope information is used to estimate the residual phase error. Together, the pupil diameter and residual phase error are used to estimate the point spread function (PSF) and compute the optimal pattern to display on the DMD. The feasibility of the proposed quality metric is verified through simulation and is further validated with results from a model eye.

\subsection{Quality Metric}

In our experimental arrangement, each $\mathrm{SH}$ focal spot is defined over a $30 \times 30$ pixel subaperture on a CCD camera and the wavefront is measured across an array of $11 \times 11 \mathrm{SH}$ lenslet subapertures. We define a quality metric to distinguish between the light coming from within the pupil and outside it. SH spots formed by light reflected from the retina through clear ocular media are more concentrated in their intensity, whereas spots formed by light scattered from other ocular structures or formed in subapertures that are not conjugated to the pupil will have a greater spread [11]. The quality metric, $Q_{M}$, is defined by the 
relation,

$$
Q_{M}(l, m)=\left\{\frac{\sum_{(i=1, j=1)}^{\left(i=N_{i}, j=N_{j}\right)} I_{F A}(i, j)-\sum_{\left(i^{\prime}=1, j^{\prime}=1\right)}^{\left(i^{\prime}=N_{i}^{\prime}, j^{\prime}=N_{j}^{\prime}\right)} I_{F W H M}\left(i^{\prime}, j^{\prime}\right)}{\sum_{(i=1, j=1)}^{\left(i^{\prime}=N_{i}^{\prime}, j^{\prime}=N_{j}^{\prime}\right)} I_{F A}(i, j)+\sum_{\left(i^{\prime}=1, j^{\prime}=1\right)} I_{F W H M}\left(i^{\prime}, j^{\prime}\right)}\right\}
$$

where, $\sum_{(i=1, j=1)}^{\left(i=N_{i}, j=N_{j}\right)} I_{F A}(i, j)$ represents the sum of intensity values within the full subaperture area (FA) defined by each lenslet, whereas $\sum_{\left(i^{\prime}=1, j^{\prime}=1\right)}^{\left(i^{\prime}=N_{i}^{\prime}, j^{\prime}=N_{j}^{\prime}\right)} I_{F W H M}\left(i^{\prime}, j^{\prime}\right)$ represents the sum of intensity values within a region that has the same width as the full width at half maximum (FWHM) of each spot. The SH subaperture index is denoted by $(l, m)$, the pixel index by $(i, j)$, and the total numbers of pixels in each column and row of the SH lenslet subaperture and the FWHM-defined subaperture are denoted by $\left(N_{i}, N_{j}\right)$ and $\left(N_{i}^{\prime}, N_{j}^{\prime}\right)$, respectively. The matrix $Q_{M}$ has approximately an inverted super-Gaussian distribution, as the more intense SH spots (within the pupil) result in a similar and lower $Q_{M}$ value and the less intense SH spots (outside the pupil) result in a comparatively higher $Q_{M}$ value that further increases towards the periphery. We select the entire $l^{\text {th }}$ row and $m^{\text {th }}$ column corresponding to the minimum value of $Q_{M}$ and then apply bottom hat filtering to distinguish between the light coming from within and outside the pupil. For each subaperture we calculate the radii from the selected row and column of the $Q_{M}$ matrix along the horizontal and vertical axes. The calculated radii along with the index of the minimum value of $Q_{M}$ as center, are used to define a circle using a least-squares fit to result in a new boundary radius $\left(r_{b}\right)$.

The equation of the single-pass PSF $[12,13]$ is given as,

$$
P S F=\left|\mathcal{F}\left\{A e^{\frac{-j 2 \pi}{\lambda} \phi(x, y)}\right\}\right|^{2}
$$

where, $\phi(x, y)$ represents the residual phase in the pupil plane, which is estimated from the measured SH slope information using a wavefront estimation algorithm [14], $A$ represents the unit amplitude transmittance having a circular aperture of diameter $2 \times r_{b}$. The residual phase errors such as those due to noise, limited closed loop bandwidth or deformable mirror stroke are not corrected by the adaptive optics system. In a double-pass AOSLO imaging system, in which the pupil size for retinal illumination and light collection is the same, the intensity distribution of the double-pass PSF is the autocorrelation of the single-pass PSF, under the assumption that the retina is a uniform diffuse reflector [13,15]. Thus, the optimum aperture pattern that needs to be displayed on the DMD is larger in size than that estimated using Eq. 2, as the size of the double-pass PSF is larger than the single-pass PSF. 


\subsection{Simulation}

We present results from a simulation to demonstrate the feasibility of the proposed pupil diameter estimation method. A super-Gaussian type intensity profile is considered in order to mimic the light reflectance through the pupil of a human eye, as shown in Fig. 1(a). The intensity distribution in the pupil is typically considered to be a super-Gaussian due to the optical Stiles-Crawford effect [16,17]. An array of $15 \times 15$ SH spots was generated using the super-Gaussian
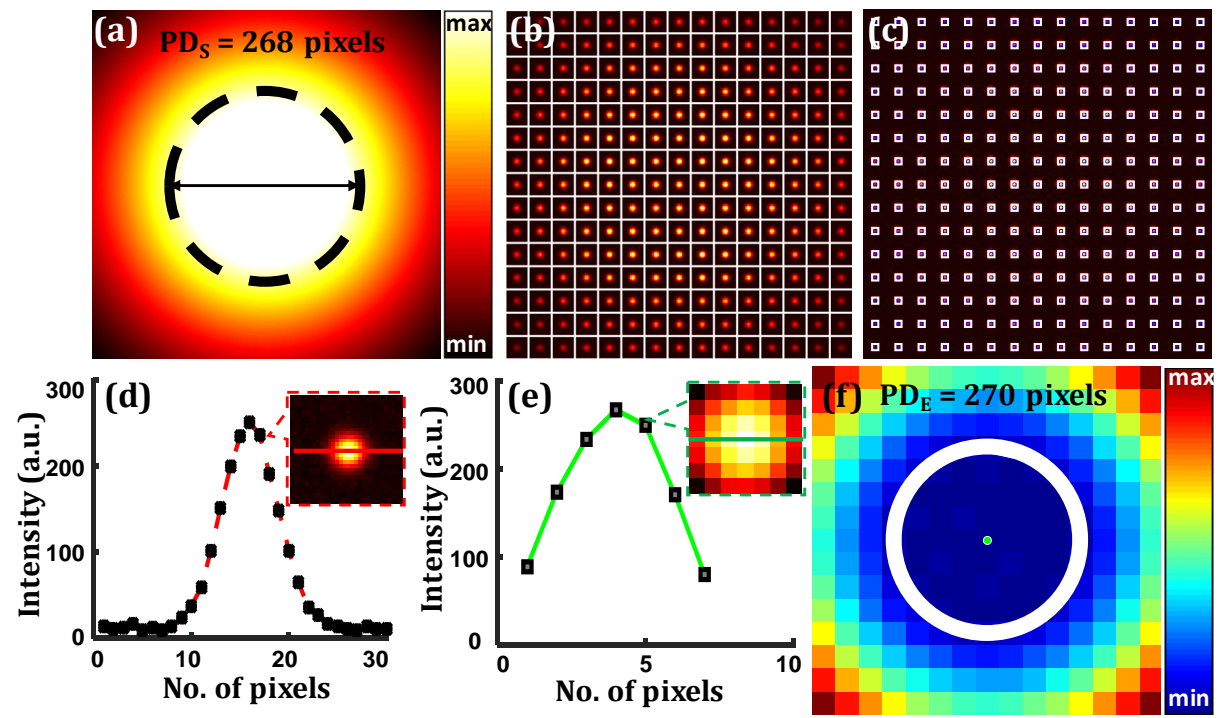

Fig. 1. False color image representing (a) a super-Gaussian intensity profile simulated with a set pupil diameter, $P D_{S}$ (black dotted circle) to obtain (b) an array of SH spots formed within SH lenslet subapertures and (c) within FWHM-defined subapertures. The intensity profile across the center of an illustrative single SH spot (inset) is shown from (d) a lenslet subaperture and from (e) a FWHM-defined subaperture, respectively so as to obtain $(\mathrm{h})$ the quality metric $Q_{M}$ and the estimated pupil diameter, $P D_{E}$ (white solid line).

pupil transmittance, defined over SH lenslet subapertures and FWHM-defined subapertures as shown in Fig. 1(b) and Fig. 1(c), respectively. In this example, the flat-top portion of the super-Gaussian is defined with 268 pixels across its diameter (the set pupil diameter, $P D_{S}$ ). The intensity profiles across the centers of an illustrative single SH spot (inset), from a lenslet subaperture and from a FWHM-defined subaperture, are shown in Fig. 1(d) and Fig. 1(e), respectively. Figure 1(f) represents the quality metric $Q_{M}$ as defined by Eq. 1. Estimated radii along the horizontal and vertical axes are obtained after bottom-hat filtering the selected $l^{t h}$ row and $m^{t h}$ column of the quality metric, $Q_{M}$. The calculated radii, along with the index of the minimum value of $Q_{M}$ as the center, are used to 
define a circle using a least-squares fit, as shown by the white solid line overlayed on the $Q_{M}$ metric, illustrated in Fig. 1(f). We take the diameter of this circle as the estimated pupil diameter $\left(P D_{E}\right)$, which is calculated to be 270 pixels. Thus, the proposed pupil diameter estimation method provides an accuracy of $99.2 \%$. Although a representative result is presented here, it is worth mentioning that we have verified the estimation of pupil diameter using the proposed quality metric for different values of $P D_{S}$. We have further analysed its working by translating the simulated super-Gaussian intensity profile in the lateral direction so as to consider the effect of eye motion in human participants. It is noticed that the average accuracy of pupil diameter estimation for different $P D_{S}$ with an array of $15 \times 15 \mathrm{SH}$ spots is $\sim 99 \%$ and it is not affected by the translation of the super-Gaussian intensity profile in the lateral direction.

\subsection{Validation with Model Eye}
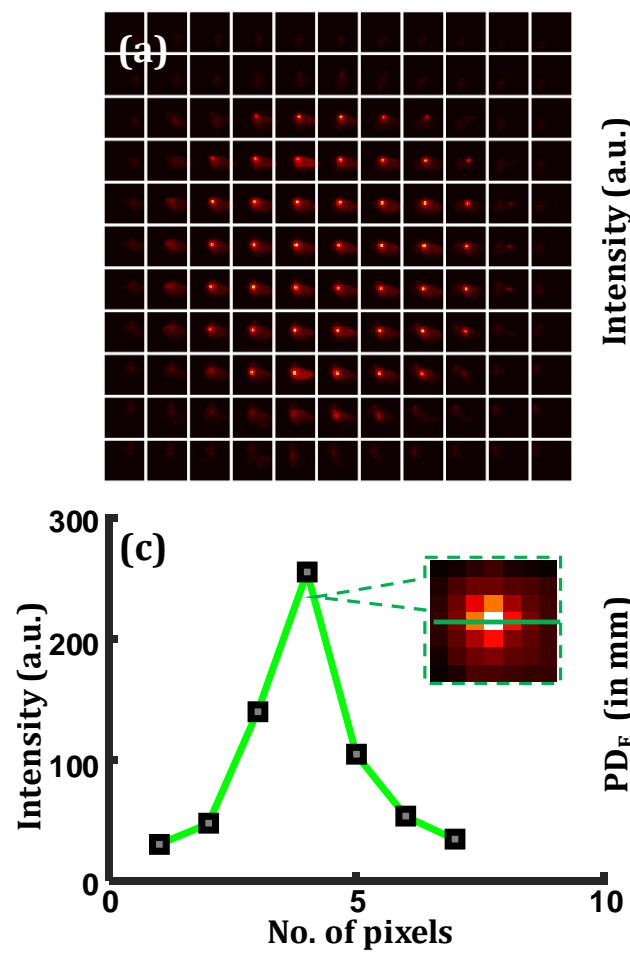
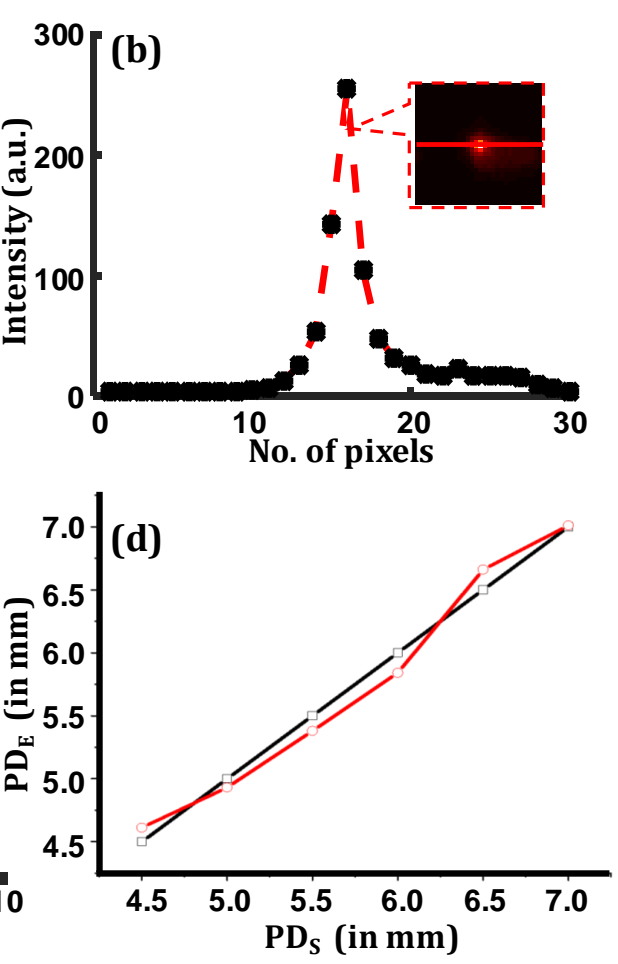

Fig. 2. (a) An array of SH spots defined for a set pupil diameter, $P D_{S}=7 \mathrm{~mm}$ and the corresponding intensity profile across the center of an illustrative single SH spot (inset) is shown from (b) a lenslet subaperture and from (c) a FWHM-defined subaperture, respectively. The (d) line plot in red show the estimated pupil diameter, $P D_{E}$ against the $P D_{S}$ for a model eye (the equality line is shown in black). 
We have further validated the performance of the pupil diameter estimation method with results from a model eye. For this purpose, the SH spots were captured for different values of $P D_{S}$ (obtained using an adjustable iris diaphragm) which ranged from $4.5 \mathrm{~mm}$ to $7 \mathrm{~mm}$ with a step size of $0.5 \mathrm{~mm}$. Figure 2(a) shows an array of SH spots for a $P D_{S}=7 \mathrm{~mm}$. Figure 2(b) and Fig. 2(c) corresponds to the intensity profiles across the centers of an illustrative single $\mathrm{SH}$ spot (inset) from a lenslet subaperture and from a FWHM-defined subaperture, respectively. The quality metric described in section 2.1 is used to estimate the pupil diameter and the line plot in red, shown in Fig. 2(d), represents the $P D_{E}$ against the $P D_{S}$ (the equality line is shown in black) and the corresponding RMSE is found to be $0.14 \mathrm{~mm}$. Thus, these results clearly establish the efficacy of the proposed pupil diameter estimation method and can be reliably used for human participants.

\section{Experimental Implementation}

The detector module of the Oxford-AOSLO system [18] was modified as shown in Fig. 3 to collect both the confocal and non-confocal light simultaneously. The

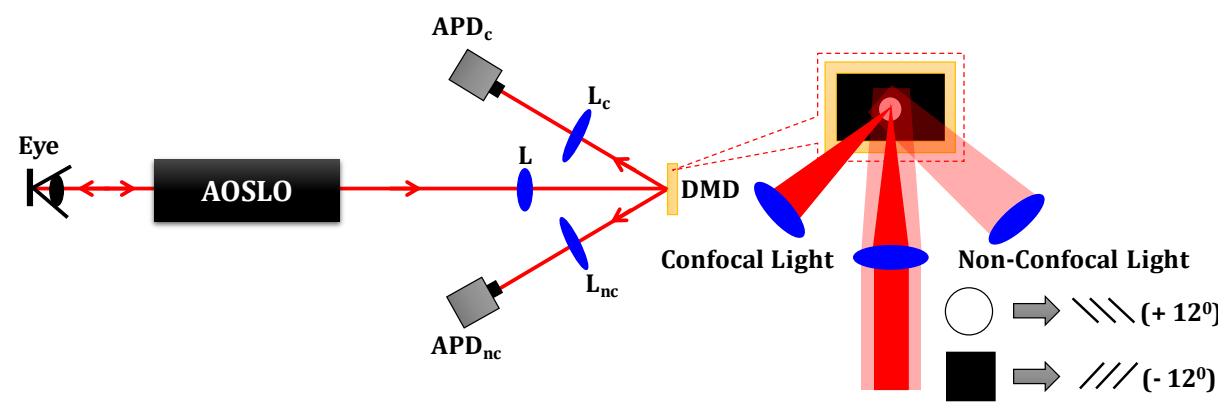

Fig. 3. Schematic of the experimental arrangement to simultaneously capture the confocal and non-confocal light using a DMD. The en-face view of the DMD panel shows the orientation of the micromirrors to $+12^{\circ}$ (for a value of 1 ) and to $-12^{\circ}$ (for a value of 0 ). A random value of 1 and 0 will orient the micromirrors to $+12^{\circ}$ and $-12^{\circ}$, respectively.

physical pinhole (placed in front of the avalanche photodiode, APD), which is conjugated to the retinal plane, was replaced with a programmable DMD that consists of $1920 \times 1080$ micromirrors with a pixel pitch of $7.56 \mu \mathrm{m}$ and a typical ON state fill factor of $94 \%$. The DMD acts as a binary device and can tilt each of its micromirrors individually to either $+12^{\circ}$ (ON state) or $-12^{\circ}$ (OFF state) around a $45^{\circ}$ axis. The plane of the DMD is kept normal to the incident beam so that the effects of diffraction from both the ON and OFF states are equivalent. A pair of identical lenses $\left(L_{c}\right.$ and $\left.L_{n c}\right)$ having a focal length of $40 \mathrm{~mm}$ and 1 
inch diameter were used to collect a majority of diffracted orders on two separate APD detectors $\left(A P D_{c}\right.$ and $\left.A P D_{n c}\right)$. A user-defined binary pattern that can be updated in real time was displayed on the DMD via a USB 2.0 interface, where a value of 1 (white) tilts the micromirrors to the ON state to collect the backscattered (confocal) light and a value of 0 (black) tilts the micromirrors to the OFF state to collect the multiply scattered (non-confocal) light, simultaneously, as shown in the en-face view of the DMD in the panel on the right side of Fig. 3. The construction of the binary aperture pattern and the calculation of the quality metric was done in Matlab (R2019b), whereas interfacing to the DMD was done using Python (Python 2.7). The current experimental arrangement is designed to collect the confocal and non-confocal light simultaneously. In this paper we investigated an annular darkfield non-confocal arrangement. However, the binary nature of the micromirrors of the DMD imposes a limitation on optimising image quality. This is because when optimising the confocal image quality by setting the required micromirrors to the $\mathrm{ON}$ state, the remaining micromirrors have to be directed towards the OFF state and that can contaminate the darkfield signal with confocal light. Thus, after deciding which micromirrors to direct light towards the confocal and darkfield detectors, we randomly orient the remaining micromirrors to reduce the contribution of confocal light entering into the darkfield detector and vice-versa. This is achieved by constructing an annular pattern with random values of 1 and 0 which will orient the micromirrors randomly in this region to $+12^{\circ}$ and $-12^{\circ}$, respectively.

\section{Results and Discussion}

We present example images from a human participant collected as part of a project that has been reviewed by and received ethics approval through the University of Oxford Central University Research Ethics Committee (R57315/RE002). The pupil diameter was estimated to be $4.80 \mathrm{~mm}$ and the FWHM of the PSF at the retinal plane to be 0.82 ADD (here ADD refers to Airy Disc Diameter given by, $\mathrm{ADD}=\frac{2.44 \times \lambda \times f}{w}$, where $f$ is the focal length of the collector lens, $\lambda$ is the wavelength of light and $w$ is the beam diameter). Figure 4(a) shows an

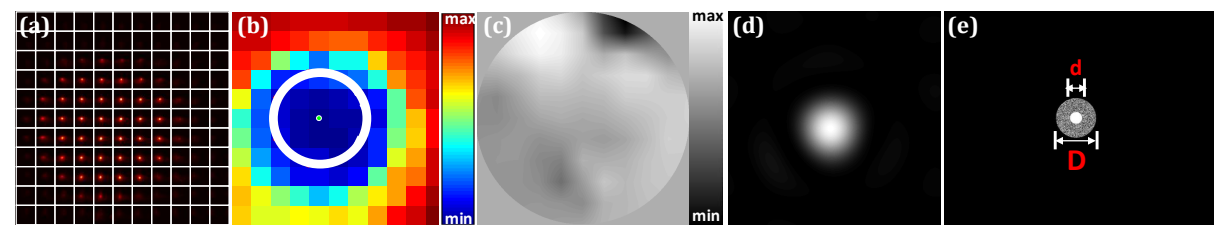

Fig. 4. (a) SH wavefront sensor image used to find (b) the quality metric and (c) the residual phase, from which $(\mathrm{d})$ the double-pass PSF is estimated. These are used to estimate (e) the optimal pattern to display on the DMD to simultaneously obtain a pair of confocal and darkfield images from a healthy human participant. We manipulate the confocal aperture diameter, d, and the darkfield inner diameter, D. 
array of SH spots, used to find the quality metric and the residual phase error as shown in Fig. 4(b) and Fig. 4(c), respectively. Based on this, the double-pass PSF is estimated as shown in Fig. 4(d) to design an optimal aperture pattern as illustrated in Fig. 4(e) to display on the DMD to simultaneously obtain confocal and darkfield images.
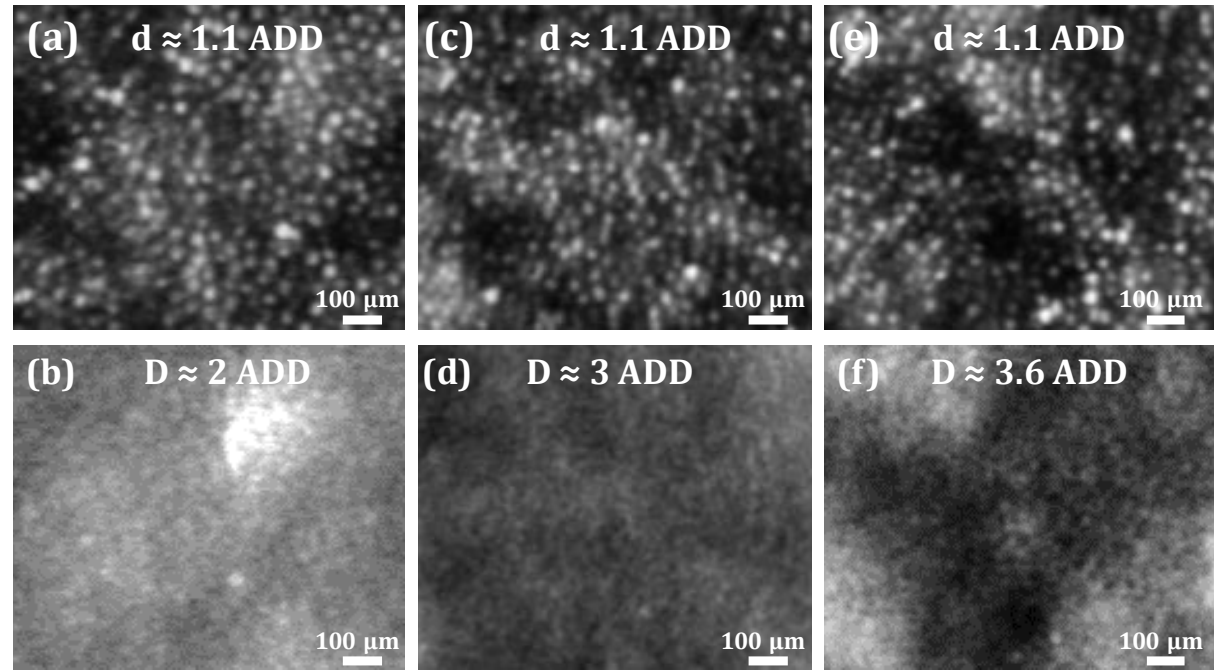

Fig. 5. A pair of simultaneous confocal and darkfield images with (a) $d=1.1$ ADD and (b) D = 2 ADD, (c) d = 1.1 ADD and (d) D = 3 ADD and (e) d = 1.1 ADD and (f) $\mathrm{D}=3.6 \mathrm{ADD}$, from a healthy human participant captured at close locations.

A number of confocal and darkfield images captured simultaneously are shown in Fig. 5. Initially, we try to obtain a good quality confocal image by setting an optimised confocal diameter (d) and then sequentially increase the darkfield inner diamter (D) until the darkfield structures are visible. There are two important factors that need to be considered to increase the visibility of darkfield structures. The first is to minimise the contribution of strong confocal light from dominating the darkfield structures and the second is to have sufficient darkfield light throughput so that signal from the darkfield structures is not swamped by noise. A pair of confocal and darkfield images captured simultaneously with $\mathrm{d}=1.1 \mathrm{ADD}$ (the optimal confocal pinhole diameter determined based on the double-pass PSF estimation) and $\mathrm{D}=2 \mathrm{ADD}$ are shown in Fig. 5(a) and Fig. 5(b), respectively. Figure 5(c) and Fig. 5(d) show the pair of confocal and darkfield images obtained with $\mathrm{d}=1.1 \mathrm{ADD}$ and $\mathrm{D}=3 \mathrm{ADD}$, respectively and Fig. 5(e) and Fig. 5(f) show the pair of confocal and darkfield images obtained with $\mathrm{d}=1.1 \mathrm{ADD}$ and $\mathrm{D}=3.6 \mathrm{ADD}$. A comparison of the confocal images (i.e., Fig. 5(a), Fig. 5(c) and Fig. 5(e)) reveal that they are of similar quality as they have the same confocal diameter, optimised based on the esti- 
mated double-pass PSF. However, a comparison of the darkfield images reveal that the darkfield structures in Fig. 5(f) are more prominent than in Fig. 5(b) or Fig. $5(\mathrm{~d})$, due to the fact that setting a larger darkfield inner diameter blocks more confocal light from entering into the darkfield detector. The randomisation of the micromirrors in the region between the confocal disc and the darkfield annulus incorporates some amount of confocal light into the darkfield image and some unwanted light into the confocal image. However, this does not degrade the image quality significantly. Although the present demonstration focuses on darkfield imaging, it can be extended to any other non-confocal imaging, such as split-detection imaging. Moreover, the current darkfield arrangement can be used to visualise the retinal pigment epithelial cells which nourish the retina and any changes in the former can lead to different eye diseases and can be a target for treatment [6].

\section{Conclusion}

In this paper, we have utilised the intensity distribution and the slope information of the $\mathrm{SH}$ spots to estimate the pupil diameter and the residual phase error, respectively. We use this information to estimate the double-pass PSF and construct an optimised aperture pattern. This reconfigurable aperture pattern, implemented with a DMD enables simultaneous optimisation of confocal and darkfield images. The method of estimation of the pupil diameter was tested in simulation and validated with a model eye. We present preliminary experimental results to demonstrate simultaneous acquisition of confocal and darkfield images in a human participant, with the potential to optimise settings independently.

\section{Acknowledgement}

Authors would like to acknowledge the financial support from various sources: Fight For Sight (1467/8); University of Oxford Wellcome Trust Institutional Strategic Support Fund (105605/Z/14/Z); the University of Oxford Medical Research Fund (MRF/LSV2015/2161); the EPA Cephalosporin Fund (CF 277); the

John Fell Oxford University Press (OUP) Research Fund (103/786 and 151/139); The Dowager Countess Eleanor Peel Trust.

\section{References}

1. Roorda, A., Romero-Borja, F., Donnelly III, W. J., Queener, H., Hebert, T. J., and Campbell, M. C.: Adaptive optics scanning laser ophthalmoscopy. Optics Express 10(9), 405-412 (2002).

2. Zhang, Y., Poonja, S., and Roorda, A.: MEMS-based adaptive optics scanning laser ophthalmoscopy. Optics Letters 31(9), 1268-1270 (2006).

3. Hammer, D. X., Ferguson, R. D., Bigelow, C. E., Iftimia, N. V., Ustun, T. E., and Burns, S. A.: Adaptive optics scanning laser ophthalmoscope for stabilized retinal imaging. Optics Express 14(8), 3354-3367 (2006). 
4. Dubra, A., Sulai, Y., Norris, J. L., Cooper, R. F., Dubis, A. M., Williams, D. R., and Carroll, J.: Noninvasive imaging of the human rod photoreceptor mosaic using a confocal adaptive optics scanning ophthalmoscope. Biomedical Optics Express 2(7), 1864-1876 (2011).

5. Burns, S. A., Elsner, A. E., Sapoznik, K. A., Warner, R. L., and Gast, T. J.: Adaptive optics imaging of the human retina. Progress in Retinal and Eye research 68, 1-30 (2019).

6. Scoles, D., Sulai, Y. N., and Dubra, A.: In vivo dark-field imaging of the retinal pigment epithelium cell mosaic. Biomedical Optics Express 4(9), 1710-1723 (2013).

7. Scoles, D., Sulai, Y. N., Langlo, C. S., Fishman, G. A., Curcio, C. A., Carroll, J., and Dubra, A.: In vivo imaging of human cone photoreceptor inner segments. Investigative Ophthalmology and Visual Science 55(7), 4244-4251 (2014).

8. Rossi, E.A., Granger, C.E., Sharma, R., Yang, Q., Saito, K., Schwarz, C., Walters, S., Nozato, K., Zhang, J., Kawakami, T. and Fischer, W.: Imaging individual neurons in the retinal ganglion cell layer of the living eye. Proceedings of the National Academy of Sciences 114(3), 586-591 (2017).

9. Chui, T. Y., VanNasdale, D. A., and Burns, S. A.: The use of forward scatter to improve retinal vascular imaging with an adaptive optics scanning laser ophthalmoscope. Biomedical Optics Express 3(10), 2537-2549 (2012).

10. Sapoznik, K. A., Luo, T., De Castro, A., Sawides, L., Warner, R. L., and Burns, S. A.: Enhanced retinal vasculature imaging with a rapidly configurable aperture. Biomedical Optics Express 9(3), 1323-1333 (2018).

11. De Castro, A., Sawides, L., Qi, X., and Burns, S. A.: Adaptive optics retinal imaging with automatic detection of the pupil and its boundary in real time using ShackHartmann images. Applied Optics 56(24), 6748-6754 (2017).

12. Chen, L., Singer, B., Guirao, A., Porter, J., and Williams, D. R.: Image metrics for predicting subjective image quality. Optometry and Vision Science 82(5), 358-369 (2005).

13. Venkateswaran, K., Roorda, A., and Romero-Borja, F.: Theoretical modeling and evaluation of the axial resolution of the adaptive optics scanning laser ophthalmoscope. Journal of Biomedical Optics 9(1), 132-139 (2004).

14. Southwell, W. H.: Wave-front estimation from wave-front slope measurements. Journal of the Optical Society of America 70(8), 998-1006 (1980).

15. Artal, P., Marcos, S., Navarro, R., and Williams, D. R.: Odd aberrations and double-pass measurements of retinal image quality. Journal of Optical Society of America A 12(2), 195-201 (1995).

16. Rativa, D., and Vohnsen, B.: Single-and multimode characteristics of the foveal cones: the super-Gaussian function. Journal of Modern Optics 58(19-20), 1809-1816 (2011).

17. Vohnsen, B.: Directional sensitivity of the retina: A layered scattering model of outer-segment photoreceptor pigments. Biomedical Optics Express 5(5), 1569-1587 (2014).

18. Young, L. K., Morris, T. J., Saunter, C. D., and Smithson, H. E.: Compact, modular and in-plane AOSLO for high-resolution retinal imaging. Biomedical Optics Express 9(9), 4275-4293 (2018). 